\title{
Prevalência de diabetes melito e fatores associados em população urbana adulta de baixa escolaridade e renda do sertão nordestino brasileiro
}

\author{
Prevalence of diabetes and associated factors in an urban adult population \\ of low educational level and income from the Brazilian Northeast wilderness
}

Ruy Lyra', Rosilda dos Santos Silva', Renan Magalhães Montenegro Jr. ${ }^{2}$, Marcus Vinicius Cardoso Matos', Nathalia Joanne Bispo Cézar', Luiz Maurício-da-Silva'

1 Departamento de Genética, Centro de Ciências Biológicas, Universidade Federal de Pernambuco

(UFPE), Recife, PE, Brasil

2 Departamento de Saúde Comunitária, Faculdade de Medicina, Universidade Federal do Ceará (UFC), Fortaleza, CE, Brasil

Correspondência para: Ruy Lyra

Av. Visconde de Albuquerque, 137 50610-090 - Recife, PE, Brasil ruylyra@smart.net.br

Recebido em 17/Mar/2010 Aceito em 6/Jul/2010

\section{RESUMO}

Introdução: O diabetes melito (DM) constitui problema de saúde pública no Brasil. Este estudo teve por objetivo investigar a prevalência de DM, sua relação com características sociodemográficas e outros fatores de risco cardiovascular em uma população adulta do sertão de Pernambuco/Brasil. Sujeitos e métodos: Foi conduzido em 2008/2009 estudo transversal na população adulta e urbana do Distrito de Canaã, município de Triunfo, sertão de Pernambuco/ Brasil. Amostra representativa constituída por 198 indivíduos com idade média de 57,7 anos foi aleatoriamente selecionada, em que $80,0 \%$ tinham apenas o ensino fundamental, e $81,3 \%$ tinham renda mensal inferior a um salário-mínimo. Resultados: Foi encontrada prevalência de $13,6 \%$ de DM e de $7,6 \%$ de disglicemia $(6,6 \%$ de glicemia de jejum alterada e 1,0\% de tolerância diminuída à glicose). Dos diabéticos, $24,0 \%$ não tinham diagnóstico. Nos diabéticos, $87,5 \%$ apresentavam síndrome metabólica (SM) e 68,0\% eram hipertensos. Houve associação positiva e significativa entre DM e IMC e entre DM e SM. Conclusão: Foi encontrada elevada prevalência de DM nessa população adulta urbana do sertão nordestino. Esses resultados assim como a sua associação com obesidade e síndrome metabólica indicam a necessidade de ações mais efetivas na prevenção dessas enfermidades. Arq Bras Endocrinol Metab. 2010;54(6):560-6

Descritores

Diabetes melito; obesidade; síndrome metabólica; escolaridade; baixa renda; prevenção; prevalência; risco cardiovascular

\section{ABSTRACT}

Background: Diabetes mellitus (DM) is a public health problem in Brazil. The objective of this study was to investigate the prevalence of DM and its relation with socio-demographic features and other cardiovascular risk factors in a adult population from the wilderness of Pernambuco, Brazil. Subjects and methods: In 2008/2009 a cross-sectional study in the adult urban population of Canãa district, city of Triunfo, in the wilderness of Pernambuco/Brazil was conducted. A representative sample of 198 individuals, with average age of 57.7 years, $80 \%$ with primary education and $81.3 \%$ with a monthly income of less than 1 minimum wage was randomly selected. Results: A prevalence of diabetes of $13.6 \%$ and $7.6 \%$ of disglycemia $(6.6 \%$ of impaired fasting glycemia, and $1.0 \%$ of impaired glucose tolerance) was found. Among those with diabetes, $24 \%$ had no prior diagnosis. Among diabetics, metabolic syndrome (MS) and arterial hypertension $(\mathrm{AH})$ were diagnosed in $87.5 \%$ and $68 \%$, respectively. Besides these, there was a positive and significant association between DM and $\mathrm{BMI}$, and between DM and MS. Conclusion: These data demonstrate a high prevalence of DM in the adult urban population from the wilderness of Pernambuco/Brazil. These results as well as their association with obesity and MS, indicate that more effective interventions are necessary for the prevention of these diseases also outside principal urban centers. Arq Bras Endocrinol Metab. 2010;54(6):560-6

\section{Keywords}

Diabetes mellitus; obesity; metabolic syndrome; education, low income; prevention; prevalence; cardiovascular risk 


\section{INTRODUÇÃO}

$\mathrm{O}$ diabetes melito tipo 2 (DM2) é uma condição grave, debilitante e que requer tratamento contínuo. É uma doença crônica cada vez mais presente, afetando aproximadamente 171 milhões de indivíduos em todo o mundo e com projeção de alcançar 366 milhões de pessoas no ano de 2030 (1). O DM2 ainda acarreta grande impacto econômico para as nações. Só nos Estados Unidos, por exemplo, os custos diretos e indiretos com a doença no ano de 2007 atingiram 174 bilhões de dólares anuais (2) e na América Latina e no Caribe no ano 2000 foram de US\$ 65 bilhões (3). A importância do diabetes se encontra, sobretudo, na perspectiva do desenvolvimento das complicações. Por sua frequência e gravidade, destacam-se as complicações crônicas. Segundo informações do Sistema Único de Saúde (SUS) do Brasil, o diabetes melito aparece como a sexta causa primária de internações hospitalares e contribui de forma significativa $(30 \%-50 \%)$ para outros fatores causais de internamento, tais como cardiopatia isquêmica, insuficiência cardíaca, colecistopatia, AVC e hipertensão arterial (HA) (4). Boletim da Organização Mundial da Saúde (OMS) para diabetes melito no Caribe e na América Latina informou que o número de mortes em 2000 foi estimado em 339.035 (3). Reduzir o impacto do DM2 significa, antes de tudo, reduzir a incidência da doença, antecipando-se ao seu aparecimento com medidas preventivas, sobretudo em indivíduos de alto risco.

Embora sabidamente crescente no mundo, pouco se conhece sobre a prevalência dessa condição em cidades que não sejam capitais brasileiras, particularmente em populações de baixa escolaridade e renda do sertão nordestino. Assim, o presente estudo teve por objetivo investigar a prevalência de DM e sua relação com características sociodemográficas e outros fatores de risco cardiovascular, em uma população urbana adulta do sertão pernambucano, onde predominam baixa escolaridade e renda. Até este momento não existem referências a estudos semelhantes nessa região.

\section{SUJEITOS E MÉTODOS}

Realizou-se em 2008/2009 um estudo transversal na população adulta (urbana) do distrito de Canaã, município de Triunfo, no sertão de Pernambuco/Brasil. Esse município está situado no sertão pernambucano, na microrregião do Pageú, a $449 \mathrm{~km}$ do Recife, capital do estado, e tem uma população de $\mathbf{1 5 . 2 2 5}$ habitantes, sendo 6.513 aqueles com 30 ou mais anos de idade
(Censo 2000 - IBGE) (5). Triunfo é a cidade mais alta do território pernambucano, situada em uma das regiões áridas nordestinas (Sertão do Pajeú). A temperatura desce com frequência a $15^{\circ} \mathrm{C}$, podendo, durante algumas ocasiões, cair para em torno de $10^{\circ} \mathrm{C}$. Diferentemente, o distrito de Canaã é situado na parte mais baixa do município de Triunfo, com isso apresentando temperaturas bem mais elevadas, características do sertão pernambucano. A sua população de 1.817 pessoas é tipicamente urbana, heterogênea e miscigenada quanto à ascendência.

O tamanho da amostra foi baseado no número de famílias residentes nesse distrito (234), sendo esta constituída por 198 indivíduos e estratificada por faixas etárias, de forma representativa da população adulta do município ( $\geq 30$ anos).

Esta pesquisa foi revisada e aprovada pelo Comitê de Ética em Pesquisa do Centro de Ciências da Saúde da Universidade Federal de Pernambuco (CEP/CCS/ UFPE) (registro $n^{\circ}$ 190/2006). Todos os participantes leram e assinaram o termo de consentimento livre e esclarecido.

Inicialmente foram identificadas e contatadas as 234 famílias residentes naquela localidade para a participação no estudo, sendo randomicamente selecionado um indivíduo de cada família. Em caso de indisponibilidade ou recusa, este foi substituído pelo familiar de idade mais próxima. Foram critérios de exclusão: gravidez, doenças consumptivas e psiquiátricas graves, retardo mental e pacientes acamados. Dos 234 indivíduos selecionados do total de famílias, 198 realizaram todas as etapas de estudo.

Os entrevistadores foram devidamente treinados $\mathrm{e}$ supervisionados por um coordenador de campo. Os participantes foram entrevistados seguindo-se um formulário padronizado cujas respostas foram registradas e codificadas com suas características sociodemográficas (sexo, idade, escolaridade, renda familiar), hábitos de vida (atividade física/sedentarismo e tabagismo) e quanto à presença de condições mórbidas prévias como DM, HA, obesidade e dislipidemia.

$\mathrm{Na}$ classificação da renda se adotou como critério o número de salários-mínimos recebidos individualmente por mês, sendo categorizados em até 1 salário-mínimo, de 1 a 2 salários-mínimos e mais de 2 salários-mínimos. Quanto à escolaridade, a estratificação baseou-se na lei de Diretrizes e Bases da Educação Nacional - Lei 9394-96 (6), que utiliza os termos ensino fundamental (equivalente ao $1^{\circ}$ grau), ensino médio (equivalente ao 
$2^{\circ}$ grau) e ensino superior. Aqueles sem qualquer escolaridade foram nominados de analfabetos. Foram ainda coletados dados quanto ao tabagismo e à atividade física. Para hábito de fumar, foram considerados tabagistas aqueles indivíduos utilizando qualquer quantidade de cigarros, excetuando-se os que nunca fumaram ou pararam de fumar há pelo menos 30 dias. $\mathrm{Na}$ avaliação da prática de atividade física e determinação de sedentarismo, foi considerada a não realização de exercícios físicos no mínimo três vezes por semana.

Após a coleta dos dados subjetivos, foram também realizadas medidas da pressão arterial $(\mathrm{PA})$, da frequência cardíaca e antropométricas dos participantes, e ao final estes foram orientados a fazer jejum de 12 horas para coleta de sangue venoso em Unidade Básica de Saúde (UBS), para a realização das determinações bioquímicas. Para a avaliação do estado de tolerância à glicose, inicialmente todos os indivíduos foram rastreados com glicemia capilar de jejum (GCJ) em campo, em dias seguintes aos da entrevista. Os diagnósticos de DM, tolerância diminuída à glicose (TDG) e glicemia de jejum alterada (GJA) foram baseados nos critérios da Sociedade Brasileira de Diabetes (7). Assim, além daqueles com diagnóstico prévio de DM2, foram considerados diabéticos aqueles com glicemia venosa de jejum $\geq 126$ $\mathrm{mg} / \mathrm{dl}$ em duas ocasiões diferentes ou glicemia 2 horas após ingestão de $75 \mathrm{~g}$ de glicose anidra no Teste Oral de Tolerância à Glicose $($ TOTG) $\geq 200 \mathrm{mg} / \mathrm{dl}$, teste este realizado em todos os que apresentaram glicemia capilar de jejum entre $100 \mathrm{mg} / \mathrm{dl}$ e $125 \mathrm{mg} / \mathrm{dl}$, bem como em 1 de cada 6 indivíduos apresentando glicemia de jejum < $100 \mathrm{mg} / \mathrm{dl}$. Naqueles com glicemia capilar $\geq 126 \mathrm{mg} / \mathrm{dl}$, o exame foi confirmado com glicemia em sangue venoso na Unidade de Saúde. Nos casos identificados de DM prévio à avaliação do controle glicêmico, foi utilizada a última glico-hemoglobina Alc (correspondente ao período do estudo), registrada no prontuário da Unidade de Saúde e obtida por revisão.

A PA foi mensurada três vezes, com intervalo mínimo de dois minutos, no braço direito de cada indivíduo, sentado, após pelo menos cinco minutos de repouso, por meio de um aparelho oscilométrico eletrônico e digital validado (Omron 705CP; Dupont, Paris, France) (8), sendo calculada a média das três medidas. O diagnóstico de HA baseou-se nos critérios das Sociedades Brasileiras de Cardiologia, de Hipertensão e de Nefrologia (9) - PA sistólica $\geq 140 \mathrm{mmHg}$ e/ou diastólica $\geq 90 \mathrm{mmHg}$, ou nos indivíduos previamente em uso de anti-hipertensivos.
Para o diagnóstico de síndrome metabólica, foram utilizados os critérios preconizados pela International Diabetes Federation (IDF) (10). Para o diagnóstico de sobrepeso ou obesidade, utilizaram-se o cálculo do índice de massa corpórea (IMC), determinado pela razão entre o peso $(\mathrm{em} \mathrm{kg})$, e o quadrado da estatura $(\mathrm{em}$ metros). Aqueles que apresentaram IMC $<18,5 \mathrm{~kg} / \mathrm{m}^{2}$ foram considerados abaixo do peso, os com IMC de 18,5 a $24,9 \mathrm{~kg} / \mathrm{m}^{2}$ foram considerados de peso normal, os com IMC de 25,0 a $29,9 \mathrm{~kg} / \mathrm{m}^{2}$ foram considerados com sobrepeso e os com IMC $\geq 30 \mathrm{~kg} / \mathrm{m}^{2}$ foram considerados obesos (11).

Nas glicosimetrias capilares, foram utilizados aparelhos portáteis Accu-Check ${ }^{\circledR}$ (Roche). As determinações sanguíneas venosas de glicose, de colesterol total, de colesterol HDL e de triglicerídeos foram realizadas por método colorimétrico (aparelho Hitachi $917^{\circledR}$ Roche).

Analisaram-se a distribuição e a homogeneidade dos grupos quanto a gênero, faixa etária, renda, escolaridade, tabagismo, sedentarismo, IMC, hipertensão arterial e síndrome metabólica.

$\mathrm{Na}$ análise estatística, foi utilizado o método do Qui-Quadrado $\left(\chi^{2}\right)$ para comparar proporções, com intervalo de confiança de $95 \%$, considerando-se um nível de significância de $5 \%(\mathrm{p}<0,05 \%)$.

\section{RESULTADOS}

Dos 198 indivíduos estudados, 68 (34,4\%) eram homens e $130(65,6 \%)$, mulheres, com idade média de 57,4 anos (31-90 anos), sendo 56,7 anos entre os homens e 57,7 anos entre as mulheres. A distribuição por faixa etária da amostra estudada de acordo com o gênero está apresentada na tabela 1 . Do total da amostra, $13,1 \%$ eram analfabetos, $80,0 \%$ tinham apenas o ensino fundamental, 6,9\% tinham ensino médio e não havia indivíduos com ensino superior. Em relação à renda mensal individual, $81,3 \%$ dos indivíduos ganhavam menos de 1 salário-mínimo, 16,7\%, entre 1 e 2 saláriosmínimos e 2,0\%, mais de 2 salários-mínimos. Quanto aos hábitos, $28,2 \%$ dos indivíduos eram fumantes e somente $27,8 \%$ praticavam atividade física.

Nesta população estudada, 27 indivíduos eram diabéticos, sendo, portanto, a prevalência de DM nessa população de 13,6\% (IC 95\%: 8,6\%-18,5\%). Esta se revelou mais elevada entre as mulheres 16,2\% (IC 95\%: 9,8\%-22,5\%), do que entre os homens avaliados - 8,8\% (IC 95\%: 1,23\%-13,7\%), com diferença significativa $(\mathrm{p}=0,01)$ (Tabela $\mathrm{l})$. 
Tabela 1. Prevalência (\%) de diabetes melito (DM) na população de Canaã/Triunfo (PE) e sua distribuição por gênero e faixa etária

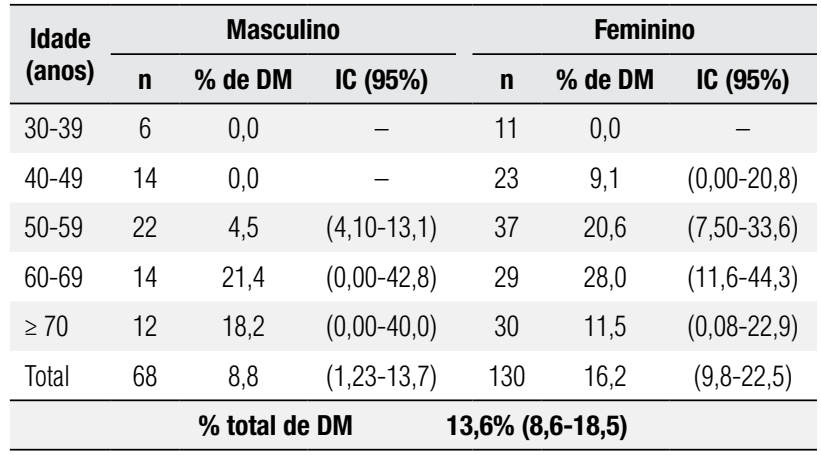

n = número absoluto de indivíduos na amostra.

Intervalo de confiança (IC) de $95 \%$.

Do total de participantes do estudo, 13 indivíduos $(6,6 \%)$ apresentaram GJA e dois $(1,0 \%)$ tinham TDG, portanto, totalizando $7,6 \%$ de casos de disglicemia ou pré-diabetes.

De todos os diabéticos identificados, 24,0\% (IC 95\%: 7,2-40,7\%) não tinham ainda diagnóstico e dos que já eram previamente diagnosticados, somente 8,0\% (IC 95\%: 0,0-18,6\%) se encontravam controlados do ponto de vista glicêmico (glico-hemoglobina Alc $<7,0 \%)$.

Verificando a distribuição desses diabéticos encontrados quanto à escolaridade, observou-se que todos os casos estavam entre analfabetos ou indivíduos com apenas o ensino fundamental. $\mathrm{O}$ mesmo não ocorreu em relação à renda, em que não foi encontrada associação entre baixa renda e a ocorrência de DM (Tabela 2).

Tabela 2. Prevalência de diabetes melito (DM) na população de Canaã/ Triunfo (PE) de acordo com a escolaridade (grau de instrução) e com a renda individual mensal

\begin{tabular}{lcccc}
\hline & $\begin{array}{c}\text { Distribuição } \\
\text { geral da } \\
\text { amostra (\%) }\end{array}$ & \% de DM & IC (95\%) & * p \\
\hline Escolaridade & & & & 0,189 \\
$\quad$ Analfabetos & 13,1 & 4,30 & $0,00-12,5$ & \\
\hline Ensino Fundamental & 80,0 & 17,1 & $10,8-23,3$ & \\
Ensino Médio & 6,9 & 0,00 & - & \\
\hline Renda mensal & & & & 0,077 \\
<1 salário-mínimo & 81,3 & 13,3 & $8,10-18,4$ & \\
1-2 salários-mínimos & 16,7 & 10,0 & $0,00-28,5$ & \\
> 2 salários-mínimos & 2,0 & 50,0 & $1,00-99,0$ & \\
\hline
\end{tabular}

${ }^{*}$ Teste $\chi^{2}$. Nível de significância de $5 \%(p<0,05 \%)$.

$\mathrm{Na}$ avaliação da relação do DM com outros fatores de risco cardiovascular estudados, não houve diferença significativa na prevalência de DM entre sedentários e praticantes de atividade física, assim como entre fumantes e não fumantes (Tabela 3 ).

No entanto, encontrou-se associação positiva e significativa entre DM e IMC, com prevalência variando de $13,2 \%$ naqueles com $\mathrm{IMC}<25 \mathrm{~kg} / \mathrm{m}^{2}$ a $17,9 \%$ nos indivíduos com IMC $\geq 30 \mathrm{~kg} / \mathrm{m}^{2}$ (Tabela 3 ).

Não houve também diferença na prevalência de diabetes entre hipertensos e não hipertensos neste estudo (Tabela 3). Todavia, 68,0\% (IC 95\%: 49,7-86,2\%) dos diabéticos detectados apresentavam HA; e, ao se estratificar os níveis pressóricos desses pacientes com DM desta amostra, observou-se que apenas $22,2 \%$ apresentavam níveis de PA nas metas recomendadas, ou seja, inferiores a $130 \times 80 \mathrm{mmHg}$. Os demais $(77,8 \%)$ apresentavam níveis anormalmente elevados de PA, sendo $18,5 \%$ entre $130 \times 80 \mathrm{mmHg}$ e $140 \times 90 \mathrm{mmHg}$ e $59,3 \%$ acima de $140 \times 90 \mathrm{mmHg}$.

A síndrome metabólica foi diagnosticada em $87,5 \%$ (IC 95\%: 74,2\%-100,0\%) dos diabéticos detectados pelo inquérito. Além disso, o DM foi significativamente mais prevalente entre os portadores de SM (Tabela 3).

Tabela 3. Prevalência de diabetes melito (DM) na população de Canaã/ Triunfo (PE) distribuída de acordo com outros fatores de risco cardiovascular (tabagismo, sedentarismo, índice de massa corpórea [IMC], hipertensão arterial e síndrome metabólica)

\begin{tabular}{|c|c|c|c|c|}
\hline & $\begin{array}{c}\text { Distribuição } \\
\text { geral da } \\
\text { amostra (\%) }\end{array}$ & $\begin{array}{c}\% \text { de } \\
\text { DM }\end{array}$ & IC (95\%) & ${ }^{\star} p$ \\
\hline Tabagismo & & & & 0,594 \\
\hline Sim & 28,2 & 12,2 & $13,0-21,3$ & \\
\hline Não & 71,8 & 15,2 & $8,90-21,4$ & \\
\hline Sedentarismo & & & & 0,564 \\
\hline Sim & 72,2 & 14,5 & $8,10-20,8$ & \\
\hline Não & 27,8 & 17,8 & $6,60-93,3$ & \\
\hline IMC (kg/m²) & & & & $<0,05$ \\
\hline$<25,0$ & 48,6 & 13,2 & $12,5-13,9$ & \\
\hline $25,0-29,9$ & 35,8 & 12,5 & $4,30-20,6$ & \\
\hline$\geq 30,0$ & 15,6 & 17,9 & $3,70-32,0$ & \\
\hline Hipertensão arterial & & & & 0,469 \\
\hline Sim & 64,1 & 13,4 & $7,40-13,9$ & \\
\hline Não & 35,9 & 9,9 & $2,30-15,6$ & \\
\hline Síndrome metabólica & & & & $<0,01$ \\
\hline Sim & 48,5 & 20,8 & $12,6-28,9$ & \\
\hline Não & 51,5 & 4,90 & $0,70-9,10$ & \\
\hline
\end{tabular}

* Teste $\chi^{2}$. Nível de significância de $5 \%(p<0,05 \%)$.

\section{DISCUSSÃO}

Este trabalho compõe um projeto amplo, cuja finalidade foi descrever aspectos epidemiológicos e genéticos 
relacionados ao DM e condições associadas da população do sertão pernambucano. Embora possivelmente não representativa da população de Triunfo, a população do distrito de Canaã foi escolhida por representar adequadamente aquela do sertão pernambucano. Esse distrito, que é situado na parte mais baixa do município e apresenta temperaturas bem mais elevadas que a média do município, se assemelha às características climáticas e socioeconômicas encontradas no restante do sertão pernambucano. Assim, os achados deste estudo são provavelmente representativos de populações urbanas de pequenos municípios, de origem miscigenada e predominantemente de baixa renda e escolaridade do sertão nordestino.

Neste, que é o primeiro analisando prevalência de DM no sertão pernambucano, a prevalência de DM correspondeu a $13,6 \%$ da população adulta do distrito de Canaã, traduzindo uma expressiva proporção de diabéticos, demonstrando que também no sertão nordestino esse é um quadro preocupante.

O número de afetados por essa doença nesta população sertaneja nordestina com 30 anos ou mais de idade foi superior ao encontrado por Malerbi e Franco (12) em estudo multicêntrico conduzido há duas décadas envolvendo várias capitais brasileiras $(7,6 \%$ dos indivíduos entre 30 e 69 anos), por Duncan e cols. (13) em Porto Alegre, RS (8,89\% dos indivíduos entre $15 \mathrm{e}$ 64 anos), por Oliveira e cols. (14) no Rio de Janeiro, RJ (7,1\% dos indivíduos entre 30 e 69 anos), por Gus e cols. (15) no estado do Rio Grande do Sul (7,0\% dos indivíduos $>20$ anos) e por Souza e cols. (16) em Campos, RJ (6,0\% dos indivíduos > 18 anos), entretanto compatível com as prevalências observadas em outros recentes estudos como o de Torquato e cols. (17) em Ribeirão Preto, SP (12,1\% dos indivíduos entre 30 e 69 anos) e de Bosi e cols. (18) em São Carlos, SP (13,5\% dos indivíduos entre 30 e 79 anos). Assim como em tais estudos, os dados do presente trabalho sinalizam para um aumento na prevalência de DM na população adulta brasileira. Não obstante, as diferenças entre as características metodológicas dos diversos inquéritos populacionais referidos impossibilitam comparações diretas entre os valores de prevalência encontrados.

Encontraram-se TDG e GJA em 1,0\% e 6,6\% dos indivíduos estudados, com um total de disglicêmicos de 7,6\%. Mesmo considerando as diferenças metodológicas dos estudos, tais frequências se aproximam dos $7,8 \%$ de intolerância à glicose encontrada no estudo multicêntrico brasileiro (12), dos 7,7\% em Ribeirão
Preto (17) e dos 5,0\% em São Carlos (18). Considerando as menores diferenças entre tais achados quando comparadas as das prevalências de DM entre os mesmos estudos, que se ressalta que foram conduzidos em diferentes décadas, se poderia especular que se devam a fatores menos modificáveis, possivelmente determinantes genéticos de (dis)função de células-beta.

A prevalência de DM neste estudo foi significativamente maior no sexo feminino, dado que, embora também encontrado por Fidelis e cols. (19), não foi observado nos estudos de Malerbi e Franco (12), Souza e cols. (16), Torquato e cols. (17), Passos e cols. (20) e Bosi e cols. (18), em que não houve diferença estatisticamente significativa entre os gêneros. No presente estudo, a prevalência do DM aumentou também com a idade, caracterizando uma curva ascendente, com relação positiva e significativa. A prevalência foi maior em homens e mulheres entre 60 e 69 anos, entretanto menor entre os com 70 anos de idade ou mais. Esse crescimento da prevalência do DM com a idade também foi encontrado por outros estudos $(12,16,17)$. A queda na faixa etária acima dos 70 anos também foi observada em homens no estudo de São Carlos, SP (18), e em ambos os sexos nos indivíduos com 80 anos ou mais no município de Teixeiras, MG (19). Na análise desses achados se deve considerar um possível viés de seleção deste estudo, em que foi obtida maior proporção de mulheres e de indivíduos com 50 anos ou mais de idade entre os entrevistados. Uma das prováveis explicações está no fato de a coleta de dados ser realizada nos domicílios dos entrevistados sem sensibilização prévia. Assim se identificou a maior dificuldade em encontrar homens e indivíduos mais jovens nos domićlios, por estes estarem muitas vezes em jornada de trabalho na ocasião das visitas.

Quanto à escolaridade, observou-se no presente estudo que todos os casos de DM estavam entre analfabetos ou indivíduos com apenas o ensino fundamental. Esses dados são concordantes com os descritos em vários outros grupos populacionais urbanos (14,16,18,21-24) que encontraram maior prevalência de diabetes entre as pessoas com menor grau de escolaridade. Entretanto, é importante salientar que, por ser uma característica marcante dessa população, quase a totalidade da amostra estava nessa condição de baixa escolaridade. Por outro lado, não foi encontrada associação entre baixa renda e a ocorrência de DM. Tal resultado é similar ao visto em outro estudo brasileiro (18), porém diferente do encontrado por Passos e cols. (20), em que houve relação inversa entre diabetes e status de 
renda familiar. Entretanto, uma vez que a maioria tinha baixa renda, esta relação necessita de confirmação em estudos delineados com esse propósito.

A obesidade é provavelmente o fator de risco mais importante para o desenvolvimento do DM tipo 2 (2527), e essa condição estava presente em $15,6 \%$ da população estudada. Contudo, quando considerados sobrepeso e obesidade, estes passam a representar a condição da maioria da população estudada $(51,4 \%)$. Vale ressaltar que se trata de uma comunidade com predomínio de baixa renda e escolaridade, o que reforça que esta é uma condição bastante prevalente também em populações menos favorecidas. No presente trabalho, encontrou-se também associação positiva e significativa entre a prevalência de DM e o IMC, com prevalência variando de $13,2 \%$ naqueles com IMC $<25 \mathrm{~kg} / \mathrm{m}^{2}$ a $17,9 \%$ nos indivíduos com IMC $\geq 30 \mathrm{~kg} / \mathrm{m}^{2}$. Tais dados se assemelham aos encontrados em outros inquéritos populacionais $(17,18,20)$.

A hipertensão arterial está presente em $20 \%$ a $60 \%$ dos indivíduos diabéticos (28), sendo uma a três vezes mais prevalente em diabéticos quando comparados a não diabéticos e a maior determinante de eventos cardiovasculares nessa população (29-31). Embora não se tenha encontrado diferença na prevalência de diabetes entre hipertensos e não hipertensos, assim como descrito em outros estudos brasileiros $(16,20,32)$, neste estudo de Canaã/Triunfo a maioria dos diabéticos apresentava HA, e, desses, somente um pequeno subgrupo encontrava-se dentro das metas recomendadas de níveis de pressão arterial iguais ou inferiores a 130 x 80 $\mathrm{mmHg}(6)$, condição que agrava o risco de inúmeros desfechos mórbidos nesses pacientes.

$\mathrm{O}$ sedentarismo tem sido associado à resistência à insulina em indivíduos não diabéticos, independentemente da obesidade (33). A prática regular de exercícios aumenta o número de capilares e fibras musculares, favorecendo a disponibilidade de glicose mediada pela insulina nessas células (34). No presente estudo, assim como no descrito por Gimeno e cols. (35), não houve diferença significativa na prevalência de DM entre sedentários e praticantes de atividade física, diferentemente Passos e cols. (20), que encontraram relação positiva entre sedentarismo e diabetes.

Vários estudos $(20,36,37)$ e uma recente metanálise (38) sugerem que o tabagismo ativo pode ser independentemente associado a DM2. Neste estudo, entretanto, não se encontrou diferença significativa na prevalência de DM entre fumantes e não fumantes.
A síndrome metabólica, além de predizer o desenvolvimento de DM2 em portadores de DM, a sua presença aumenta consideravelmente o risco de doenças cardiovasculares (DCVs) (39-40). De forma concordante, no presente estudo, o DM foi significativamente mais prevalente entre os portadores de SM, além de essa condição ter sido identificada na maioria extrema $(87,5 \%)$ dos diabéticos detectados pelo inquérito.

Em conclusão, este estudo revelou elevada prevalência de DM entre os residentes do distrito de Canaã, região urbana do município de Triunfo, no sertão de Pernambuco, em população caracterizada por baixas escolaridade e renda, sendo significativamente mais presente em mulheres, nos indivíduos com o IMC superior a 25 $\mathrm{kg} / \mathrm{m}^{2}$ e com a presença de SM. Esses resultados corroboram os achados de elevada e possivelmente crescente prevalência em vários municípios brasileiros e indicam a necessidade de intervenções para detecção precoce $\mathrm{e}$ controle adequado do DM2 e maior atenção quanto a tal enfermidade e suas comorbidades nessa camada social. Ações promotoras de saúde efetivas são necessárias, com $\mathrm{o}$ intuito de reduzir os riscos associados de desfechos desfavoráveis e seu impacto social. O aprofundamento desse assunto, por meio de novos inquéritos epidemiológicos com ênfase nos aspectos nutricionais, estilo e qualidade de vida dessa população, além da avaliação de componente(s) genético(s), possivelmente poderá esclarecer esses achados nos residentes do sertão nordestino.

Agradecimentos: Os autores agradecem à Prefeitura de Triunfo, por intermédio do seu prefeito Luciano Bonfim e equipe de saúde (Secretário, Enfermeiras e Agentes de Saúde), pela colaboração no desenvolvimento deste estudo. Também agradecem à Fundação de Amparo à Ciência e Tecnologia do Estado de Pernambuco e ao Conselho Nacional de Desenvolvimento Científico e Tecnológico (CNPq) (processo PPSUS - APQ-0054-2.02/07 EDITAL: MS/ CNPq/FACEPE - 09/2006) pelo financiamento desse estudo e fomento à pesquisa, bem como ao Laboratório Roche, pela doação das tiras reagentes para determinações de glicemias capilares.

Vinculação acadêmica: Este artigo é parte da tese de doutorado de Ruy Lyra da Silva Filho pelo Programa de Pós-Graduação em Genética e Biologia Molecular da Universidade Federal de Pernambuco (UFPE).

Declaração: os autores declaram não haver conflitos de interesse científico neste estudo.

\section{REFERÊNCIAS}

1. Wild S, Roglic G, Green A, Sicree R, King H. Global prevalence of diabetes: estimates for the year 2000 and projections for 2030. Diabetes Care. 2004;27(5):1047-53.

2. American Diabetes Association. Economic costs of diabetes in U.S. in 2007. Diabetes Care. 2008;31(3):596-615. 
3. Barceló A, Aedo C, Rajpathak S, Robles S. The cost of diabetes in Latin America and the Caribbean. Bull World Health Organ. 2003;81(1):19-27.

4. Silvestre J. Hospitalizações SUS 1997. Coordenadoria da Atenção à Saúde do Idoso. Ministério da Saúde; 1997.

5. Instituto Brasileiro de Geografia e Estatística. (IBGE). [Acesso em 2009 jul 20]. Disponível em: http://www.ibge.gov.br.

6. Brasil. Lei $n^{\circ}$ 9.394, de 20 de dezembro de 1996. Estabelece as diretrizes e bases da Educação Nacional. Diário oficial da República Federativa do Brasil. Brasília; 1996.

7. Sociedade Brasileira de Diabetes. Tratamento e acompanhamento do diabetes mellitus: Diretrizes da Sociedade Brasileira de Diabetes. [Acesso em: 2009 nov 5] Disponível em: http://www. diabetes.org.br/educacao/docs/diretrizes.pdf.

8. O'Brien E, Mee F, Atkins N, Thomas M. Evaluation of three devices for self measurement of blood pressure according to the revised British Hypertension Society Protocol: the Omron HEM-705CP, Philips HP5332, and Nissei DS-175. Blood Press Monit. 1996;1:55-61.

9. Sociedade Brasileira de Cardiologia-SBC, Sociedade Brasileira de Hipertensão-SBH e Sociedade Brasileira de Nefrologia-SBN. V Diretrizes Brasileiras de Hipertensão Arterial. Arq Bras Cardiol. 2007;89(3):e24-79.

10. The IDF consensus worldwide definition of the metabolic syndrome [Acesso em: 2009 nov 5]. Disponível em: http://www.idf.org/ webdata/docs/IDF_Metasyndrome_definition.pdf

11. World Health Organization. WHO Expert Committee on Physical Status: the use and interpretation of anthropometry phisical status. Geneva: World Health Organization; 1995. (WHO Technical Report Series, vol. 854)

12. Malerbi D, Franco LJ. Multicenter study of the prevalence of diabetes mellitus and impaired glucose tolerance in the urban Brazilian population aged 30-69 years. The Brazilian Cooperative Group on the Study of Diabetes Prevalence. Diabetes Care. 1992;15(11):1509-16.

13. Duncan BB, Schmidt MI, Polanczyk CA, Homrich CS, Rosa RS, Achutti AC. Risk factors for non-communicable diseases in a metropolitan area in south of Brazil: prevalence and simultaneity. Rev Saude Publica. 1993;27(1):43-8.

14. Oliveira JE, Milech A, Franco LJ.The prevalence of diabetes in Rio de Janeiro, Brazil. The Cooperative Group for the Study of Diabetes Prevalence in Rio de Janeiro. Diabetes Care. 1996;19(6):663-6.

15. Gus I, Fischmann A, Medina C. Prevalence of risk factors for coronary artery disease in the Brazilian State of Rio Grande do Sul. Arq Bras Cardiol. 2002;78(5):478-90.

16. Souza LJ, Chalita FEB, Reis AF, Teixeira CL, Gicovate Neto C, Bastos DA, et al. Prevalência de diabetes mellitus e fatores de risco em Campos dos Goytacazes, RJ. Arq Bras Endocrinol Metab. 2003;47(1):69-74.

17. Torquato MT, Montenegro Júnior RM, Viana LA, de Souza RA, Lanna CM, Lucas JC, et al. Prevalence of diabetes mellitus and impaired glucose tolerance in the urban population aged 30-69 years in Ribeirão Preto (São Paulo), Brazil. Sao Paulo Med J. 2003;121(6):224-30.

18. Bosi PL, Carvalho AM, Contrera D, Casale G, Pereira MA, Gronner MF, et al.. Prevalência de diabetes melito e tolerância à glicose diminuída na população urbana de 30 a 79 anos da cidade de São Carlos, São Paulo. Arq Bras Endocrinol Metabol. 2009;53(6):726-32.

19. Fidelis LC, Moreira OC, Teodoro BG, Oliveira CEP. Prevalência de diabetes mellitus no município deTeixeiras-MG. Rev Bras Ativ Fís Saúde. 2009;14(1):23-7.

20. Passos VM, Barreto SM, Diniz LM, Lima-Costa MF. Type 2 diabetes: prevalence and associated factors in a Brazilian community - The Bambuí health and aging study. Sao Paulo Med J. 2005;123(2):66-71.
21. Barceló A, Rajpathak S. Incidence and prevalence of diabetes mellitus in the Americas. Rev Panam Salud Publica. 2001;10(5):300-8.

22. Maty SC, Everson-Rose SA, Haan MN, Raghunathan TE, Kaplan GA. Education, income, occupation, and the 34-year incidence (1965-99) of type 2 diabetes in the Alameda County Study. Int J Epidemiol. 2005;34(6):1274-81.

23. Espelt A, Borrell C, Roskam AJ, Rodríguez-Sanz M, Stirbu I, Dalmau-Bueno $A$, et al. Socioeconomic inequalities in diabetes mellitus across Europe at the beginning of the 21st century. Diabetologia. 2008;51(11):1971-9.

24. Ong KL, Cheung BM, Wong LY, Wat NM, Tan KC, Lam KS. Prevalence, treatment, and control of diagnosed diabetes in the U.S. National Health and Nutrition Examination Survey 1999-2004. Ann Epidemiol. 2008;18(3):222-9.

25. Gigante DP, Barros FC, Post CL, Olinto MT. Prevalência de obesidade em adultos e seus fatores de risco. Rev Saude Publica. 1997;31(3):236-46.

26. Berber A, Gómez-Santos R, Fanghänel G, Sánchez-Reyes L. Anthropometric indexes in the prediction of type 2 diabetes mellitus, hypertension and dyslipidaemia in a Mexican population. Int J Obes Relat Metab Disord. 2001;25(12):1794-9.

27. VisscherTL, Seidell JC. The public health impact of obesity. Annu Rev Public Health. 2001;22:355-75.

28. American Diabetes Association. Standards of medical care in diabetes. Diabetes Care. 2005;28:S4-36.

29. Wingard D, Barrett-Connor E. Heart disease and diabetes. In: Diabetes in America. Washington DC, US. Govt Printing Office; 1995. p. $429-48$.

30. Sowers JR, Epstein M, Frohlich ED. Diabetes, hypertension, and cardiovascular disease: an update. Hypertension. 2001;37:1053-9.

31. Bakris GL, Williams M, Dworkin L, Elliott WJ, Epstein M, Toto R, et al. National Kidney Foundation Hypertension and Diabetes Executive Committees Working Group. Preserving renal function in adults with hypertension and diabetes: a consensus approach. Am J Kidney Dis. 2000;36:646-61.

32. Barbosa JB, Silva AA, Santos AM, Monteiro Júnior FC, Barbosa MM, Barbosa MM, et al. Prevalência da hipertensão arterial em adultos e fatores associados em São Luís-MA. Arq Bras Cardiol. 2008;91(4):260-6.

33. Mayer-Davis EJ, D'Agostino R Jr, Karter AJ, Haffner SM, Rewers MJ, Saad M, et al. Intensity and amount of physical activity in relation to insulin sensitivity: the Insulin Resistance Atherosclerosis Study. JAMA. 1998;279(9):669-74.

34. Utriainen $T$, Holmäng $A$, Björntorp $P$, Mäkimattila $S$, Sovijärvi $A$, Lindholm $\mathrm{H}$, et al. Physical fitness, muscle morphology, and insulin-stimulated limb blood flow in normal subjects. Am J Physiol. 1996;270(5 Pt 1):E905-11.

35. Gimeno SG, Ferreira SR, Franco LJ, Hirai AT, Matsumura L, Moisés RS. Prevalence and 7-year incidence of type II diabetes mellitus in a Japanese-Brazilian population: an alarming public health problem Diabetologia. 2002;45(12):1635-8.

36. Eliasson B. Cigarette smoking and diabetes. Prog Cardiovasc Dis. 2003;45(5):405-413

37. Chiolero A, Faeh D, Paccaud F, Cornuz J. Consequences of smoking on body weight, body fat distribution, and insulin resistance: narrative review. Am J Clin Nutr. 2008;87(4):801-9.

38. Willi C, Bodenmann P, Ghali WA, Faris PD, Cornuz J. Active smoking and the risk of type 2 diabetes: a systematic review and meta-analysis. JAMA. 2007;12;298(22):2654-64.

39. Laaksonen DE, Lakka HM, Niskanen LK, Kaplan GA, Salonen JT, Lakka TA. Metabolic syndrome and development of diabetes mellitus: application and validation of recently suggested definitions of the metabolic syndrome in a prospective cohort study. Am J Epidemiol. 2002;1;156(11):1070-7.

40. Eckel RH, Grundy SM, Zimmet PZ. The metabolic syndrome. Lancet. $2005 ; 365(9468): 1415-28$ 